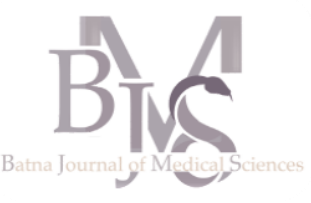

Etude d'évaluation du " Poids Sec » du patient hémodialysé chronique dans le Service de Néphrologie et d'hémodialyse du CHU du Point G (A propos de 40 cas).

\title{
Evaluation study of the "Dry Weight" of the chronic hemodialysis patient in the Department of Nephrology and Hemodialysis, CHU Point G (Report of 40 cases).
}

\author{
Alhadji Ahmadou Tounkara ${ }^{1}$, Nouhoum Coulibaly ${ }^{1}$, Idrissa Sissoko ${ }^{2}$, Lanseni Keita ${ }^{3}$; \\ Saharé Fongoro ${ }^{1}$, Mahamane Kalil Maiga ${ }^{1}$
}

${ }^{1}$ Service de Néphrologie et d'hémodialyse du CHU Point G. Bamako - Mali

${ }^{2}$ Service d'Urologie du CHU du Point G. Bamako - Mali

${ }^{3}$ Service de Cardiologie du CHU du Point G. Bamako - Mali

\section{Correspondance à :}

Alhadji Ahmadou TOUNKARA

tounka79@yahoo.fr

DOI :https://doi.org/10.48087/ B]MSoa.2016.3210

Il s'agit d'un article en libre accès distribué selon les termes de la licence Creative Commons Attribution International License (CC BY 4.0), qui autorise une utilisation, une distribution et une reproduction sans restriction sur tout support ou format, à condition que l'auteur original et la revue soient dûment crédités.

\section{RÉSUMÉ}

But : évaluer le « Poids Sec » des patients hémodialysés chroniques du service, utilisant nos propres critères. Matériels et Méthodes : il s'agit d'une étude prospective réalisée dans le service de Néphrologie et d'hémodialyse du CHU du Point G du 10 Mars 2010 au 10 février 2011 incluant 40 patients hémodialysés chroniques. Nous avions prédéfini nos critères d'obtention de «Poids sec»: critères A: absence d'HTA entre les séances d'hémodialyse, absence de cardiomégalie radiologique, pas de signe de surcharge hydrosodée cliniquement ou radiologiquement décelable, pas de prise de médicament antihypertenseur; critères B : présence d'HTA entre les séances d'hémodialyse voire une PA normale sous médicaments antihypertenseurs, absence de cardiomégalie, absence de signe de surcharge hydrosodée cliniquement ou radiologiquement décelable. Résultats : quarante patients avaient participé à l'étude ; leur âge moyen était de 45,12 $\pm 17,50$ ans, pour une durée moyenne en dialyse de $3,25 \pm 1,52$ ans. Les hommes représentaient $57,5 \%$ des patients. Les étiologies étaient dominées par les néphropathies vasculaires $(32,5 \%)$ suivies des glomérulonéphrites chroniques $(27,5 \%)$ et des néphrites interstitielles chroniques $(15 \%)$. La moyenne de prise de poids interdialytique était de $3,08 \mathrm{~kg}$ et $97,5 \%$ des patients étaient oligoanuriques. Les patients ayant atteint leur poids sec selon nos " critères A » ou " critères B » représentaient respectivement $17,5 \%$ et $55 \%$ des cas. Les patients n'ayant pas atteint leur poids sec selon nos « critères " représentaient 27,5\%. Conclusion : l'établissement du « Poids sec » du malade hémodialysé chronique reste encore difficile. Des facteurs autres que la prise de poids interdialytique ou l'ultrafiltration méritent d'être considérés.

Mots-clés: Poids sec, Prise de poids interdialytique, Ultrafiltration, Mali.

\begin{abstract}
Purpose: to evaluate the "dry weight" of our chronic hemodialysis patients, according to our own definitions. Materials and Methods: This was a prospective study in the Nephrology Department and hemodialysis, CHU Point G between March 10, 2010 and February 10, 2011 including 40 chronic hemodialysis patients. We had our pre-defined criteria for obtaining "dry weight": criteria A: absence of hypertension between hemodialysis sessions, no radiological cardiomegaly, no signs of fluid overload, clinically or radiologically detectable, not taking antihypertensive medication; and criteria B: presence of hypertension between hemodialysis sessions even under normal BP antihypertensive drugs, absence of cardiomegaly, no fluid overload sign, clinically or radiologically detectable. Results: Forty patients participated in the study. Their average age was $45.12 \pm$ 17.50 years, for an average dialysis time of $3.25 \pm 1.52$ years. Men accounted for $57.5 \%$ of patients. The etiologies were dominated by vascular nephropathy $(32.5 \%)$ followed by chronic glomerulonephritis $(27.5 \%)$ and chronic interstitial nephritis (15\%). The average interdialytic weight gain was of $3,08 \mathrm{~kg}$ and $97.5 \%$ of patients were oligo-anuric. Patients who have reached their dry weight as our "Criteria $A$ " or our "Criteria B" accounted respectively for $17.5 \%$ and $55 \%$ of cases. Patients who have not reached their dry weight as any of our "criteria" represented $27.5 \%$. Conclusion: establishing the "dry weight" of chronic hemodialysis patients remains difficult. Factors other than taking interdialytic weight or ultrafiltration merit consideration.
\end{abstract}

Keywords: Dry weight; interdialytic Weight Gain; Ultrafiltration; Mali.

\section{دراسة تقييمية ل" الوزن الجاف " لمرضى غسيل الكلى المزمن في مصلحة أمراض وغسيل الكلى في}

المستثفى الجامعي نقطة ج (سرد 40 حالئ)

Pour citer l'article :

Tounkara AA, Coulibaly N,

Sissoko I, et al. Etude

d'évaluation du « poids

sec » du patient

hémodialysé chronique

dans le service de

Néphrologie et

d'Hémodialyse du CHU du point $\mathrm{G}$ (A propos de 40 cas). Batna J Med Sci 2016;3(2):104-107.

https://doi.org/10.48087/ BJMSoa.2016.3210

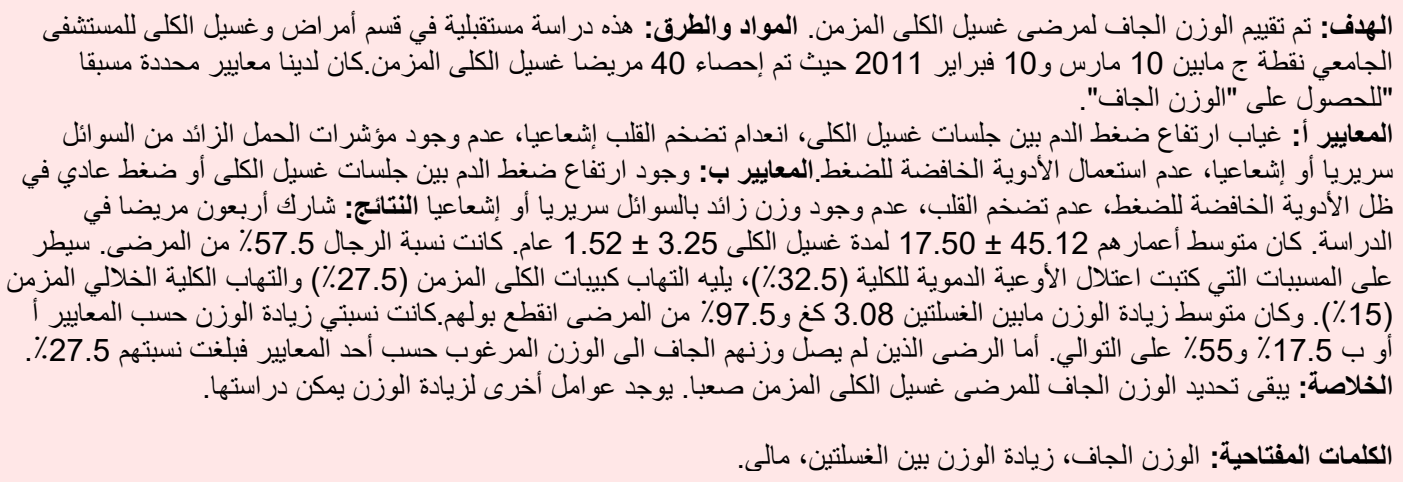




\section{INTRODUCTION}

Le concept de «Poids sec» fut introduit dans la pratique néphrologique avec l'avènement de l'hémodialyse chronique [1]. Il désigne le poids du patient en fin de séance de dialyse pour lequel la volémie plasmatique est proche de la normale. En dépit de son caractère dynamique et malgré l'absence de critères cliniques ou paracliniques définitifs pour son établissement, la recherche d'un «Poids sec» s'avère nécessaire afin d'éviter les complications de la surcharge hydrosodée ou d'une ischémie aigue secondaire à une ultrafiltration (UF) trop rapide [2,3].

Plusieurs paramètres sont utilisés dans le cadre de l'évaluation du «Poids sec», à savoir [4]:

La Pression artérielle (PA) : partant du principe que l'HTA de l'hémodialysé résulte dans $80 \%$ des cas d'une surcharge hydrosodée, l'HTA serait la traduction d'une surcharge hydrosodée alors que l'hypotension traduit une déplétion du secteur extracellulaire (SEC) [5]. Les limites de ce paramètre résultent du fait que certains patients en inflation hydrique restent normotendus (hypo albuminémie majeure, insuffisance cardiaque) alors que d'autres, quoiqu'en euvolémie ou déshydratés restent hypertendus ;

La mesure du diamètre de la veine cave inferieure (VCI) et la pression dans l'oreillette droite (OD) par échographie: ici, le principe est basé sur une relation censée linéaire entre le diamètre de la VCI et celui de l'OD définissant ainsi [6] l'hyperhydratation comme une pression dans l'OD $>7 \mathrm{mmHg}$ associée à une augmentation du diamètre de la VCI $\geq 11,5$ $\mathrm{mm} / \mathrm{m}^{2}$; alors que la déshydratation associe une pression dans l'OD $<3 \mathrm{mmHg}$ à un diamètre de la $\mathrm{VCI}<8 \mathrm{~mm} / \mathrm{m}^{2}$. Les limites de cette approche résident dans le fait qu'elle n'est pas applicable en cas d'insuffisance cardiaque droite (ICD) ou d'insuffisance tricuspidienne, ou en cas de déséquilibre des fluides de part et d'autre des capillaires ou en cas de syndrome de fuite capillaire comme la septicémie ;

Le dosage plasmatique de l'Arterial Natriuretic Peptide (ANP) et du Cyclic Ghanosine Monophosphate (GNC) [1] : l'ANP est une hormone vasodilatatrice et natriurétique secrétée par les cellules atriales et auriculaires en réponse à une augmentation de la volémie plasmatique. Certes des taux plus élevés d'ANP ont été observés chez des patients dialysés en surcharge hydrosodée juste avant les séances de dialyse avec une baisse significative de ce taux sans revenir à la normale juste après les séances de dialyse. Les limites de cette approche restent le grand âge, le taux de vasopressine et de noradrénaline voire la présence d'insuffisance mitrale. Les mêmes constats sont valables pour le GNC dont la sécrétion est corrélée à celle de l'ANP.

La régulation du volume sanguin basée sur le rétrocontrôle, la modification de la conductivité et le taux d'ultrafiltration (UF) : cette approche consiste à prescrire une courbe de réduction $\mathrm{du}$ volume sanguin modifiable à tout instant selon le niveau de remplissage vasculaire pendant la séance de dialyse. La persistance d'un remplissage vasculaire après la suspension de l'UF équivaut à un poids sec haut et l'inverse correspond à un poids sec trop bas. Cette technique reste très prometteuse. Elle est limitée par la durée très courte des séances conventionnelles d'hémodialyse soit 4 heures.

L'impédance: c'est une technique basée sur le suivi des volumes hydriques de l'organisme estimés à partir de l'émission des fréquences nulles et infinies et le calcul des résistances tout en tenant compte de la présence d'éléments conducteurs et non conducteurs. A cela s'ajoute des mesures d'hématocrite par méthode optique et le remplissage vasculaire en temps réel. Ceci permet l'ajustement de l'UF au remplissage vasculaire jusqu'à atteindre le poids optimal du patient. La limite de cette technique reste là encore le temps imparti pour la séance de dialyse généralement trop court (04h), sans compter son coût.

\section{PATIENTS ET MÉTHODES}

Il s'agissait d'une étude prospective, réalisée dans le service de Néphrologie et d'Hémodialyse du CHU du Point G du 10 Mars 2010 au 10 février 2011 incluant 40 patients hémodialysés chroniques sur 90 que comptait le centre à l'époque.

Etaient inclus dans l'étude, les patients hémodialysés chroniques du service pendant ladite période, participant régulièrement à leurs séances d'hémodialyse et ayant accepté d'y adhérer librement après un consentement éclairé. Les dialysés chroniques en séjours dans le service, les cas d'insuffisance rénale aigue dialysés et les dialysés chroniques du service n'ayant pas accepté de participer à l'étude y ont été exclus. Tous les patients recevaient 2 séances hebdomadaires de dialyse d'une durée de 5 heures chacune.

Sur le plan clinique, les mesures de la TA, de la diurèse résiduelle, du poids et la recherche de signes de surcharge hydrique ou de déshydratation ont été effectuées avant et après chaque séance de dialyse et ce pendant 12 séances. En plus, les étiologies, la durée en hémodialyse et l'âge des patients ont été relevé. Sur le plan paraclinique, nous avons demandé une NFS et une radiographie thoracique, afin de rechercher une éventuelle pleurésie, une cardiomégalie ou des œdèmes d'origine anémique.

Nos critères d'obtention de «Poids sec» étaient:

- Critères $A$ : absence d'HTA entre les séances d'hémodialyse, absence de cardiomégalie radiologique, pas de signe de surcharge hydrosodée cliniquement ou radiologiquement décelable, pas de prise de médicament antihypertenseur entre les séances d'hémodialyse ;

- Critères $B$ : présence d'HTA entre les séances d'hémodialyse voire une PA normale sous médicament antihypertenseur, absence de cardiomégalie, absence de surcharge hydrosodée cliniquement ou radiologiquement décelable.

Toutes les données ont été relevées sur des fiches individuelles, saisies à partir du logiciel Microsoft World 2010, analysées avec le logiciel SPSS version 12.0 .

\section{RÉSULTATS}

$\mathrm{Au}$ total, 40 patients avaient participé à l'étude. La moyenne d'âge des patients était de 45,12 $\pm 15,70$ ans pour des extrêmes de 23 et 77ans. Les hommes représentaient 57,5 \% avec un ratio de 1,35 en leur faveur. L'accès vasculaire était de type fistule artério-veineuse (FAV) dans $90 \%$ des cas, le cathéter fémoral et jugulaire étaient respectivement utilisés chez $7,5 \%$ et $2,5 \%$ des patients. La durée moyenne des patients en hémodialyse était de $3,25 \pm 1,52$ ans pour des extrêmes de 10 mois et de 7 ans.

Les étiologies retrouvées étaient: les néphropathies vasculaires (NV) 32,5\%, les glomérulonéphrites chroniques (GNC) 27,5\%, les Néphrites interstitielles chroniques (NIC) $15 \%$, les néphropathies indéterminées (NI) 10\%, la 
néphropathie diabétique (ND) 7,5 \%, la polykystose rénale(PKR), la néphropathie associée au VIH (HIVAN) et l'insuffisance rénale rapidement progressive (IRRP) respectivement retrouvées à 2,5\%. Sur le plan diurèse résiduelle, les anuriques et les oliguriques représentaient respectivement $82,5 \%$ et $15 \%$ des patients. Seul un patient avait une diurèse normale soit $2,5 \%$.

La moyenne de taux d'Hb était de 8,2 g/dl (extrêmes 6,4 et $11,2 \mathrm{~g} / \mathrm{dl}$ ) et $92,5 \%$ des patients avaient un taux d'Hb $\leq$ $10 \mathrm{~g} / \mathrm{dl}$. La radiographie thoracique de face était normale dans $72,5 \%$, une cardiomégalie était retrouvée dans $27,5 \%$ des cas.

Les différents types de surcharge hydriques retrouvées étaient: les œdèmes des membres inférieurs (OMI $=30 \%)$, les œdèmes du visage $(7,5 \%)$, l'ascite $(2,5 \%)$ et la pleurésie $(2,5 \%)$. La moyenne de prise de poids interdialytique(PPID) était 3,08 $\mathrm{kg}$ (extremes 1 et $5 \mathrm{~kg}$ ), pour une moyenne d'UF de 2,96 L (extrêmes 1 et $5 \mathrm{~L}$ ).

La pression artérielle moyenne d'entrée (PAM/E) était de 133/84 mm hg, 35,5\% des patients étaient normotendus, l'HTA grade 1,2 et 3 était respectivement retrouvées chez $27,5 \% ; 20 \%$ et $15 \%$ des patients. La pression artérielle moyenne de sortie (PAM/S) était de 129/81 mm hg, avec $42,5 \%$ de patients normotendus. L'HTA grade 1, 2 et 3 était respectivement retrouvée chez $30 \% ; 15 \%$ et $5 \%$ des patients. L'hypotension et les crampes musculaires étaient retrouvées chez 2 patients, soit $5 \%$.

Selon nos critères d'obtention de poids sec, $17,5 \%$ des patients répondaient aux critères $A, 55 \%$ des patients répondaient aux critères $B$ et ceux ne répondant ni aux critères A ou B représentaient $27,5 \%$ des patients (tableau 1 ).

\section{DISCUSSION}

Au total, 40 patients avaient participé dans notre étude ; leur moyenne d'âge était de 45,12 $\pm 15,70$ ans (extrêmes de 23 et 77 ans) et les hommes représentaient $57,5 \%$. Cet âge moyen de nos patients était en dessous de celui observé en occident où les patients étaient en moyenne dans la 6ème décade, mais nos données relatives à la prédominance masculine étaient proches de celles décrites dans la littérature $[5,7,9]$.

La durée moyenne de nos patients en dialyse était de 3,25 \pm 1,52 ans, et les étiologies étaient dominées par les néphropathies vasculaires $(32,5 \%)$ suivies des glomérulonéphrites chroniques $(27,5 \%)$ et des Néphrites interstitielles chroniques (15\%). L'oligurie et la diurèse normale étaient respectivement retrouvées chez $82,5 \%$ et $2,5 \%$ de nos patients.

La durée en dialyse est généralement associée à une réduction de la diurèse. En effet, plus les patients durent en hémodialyse, moins leur diurèse résiduelle est conséquente. Cette diurèse résiduelle est un élément capital d'orientation dans la détermination du régime diététique individualisé du patient dialysé. Des facteurs comme la durée en dialyse et la néphropathie sous-jacente peuvent jouer sur cette diurèse résiduelle, c'est le cas souvent des NIC avec perte de sel.

Le taux moyen d'Hb de nos patients était de $8,2 \mathrm{~g} / \mathrm{dl}$ (extrêmes 6,4 et 11,2 ) et $92,5 \%$ des patients avaient un taux $\mathrm{d}^{\prime} \mathrm{Hb} \leq 10 \mathrm{~g} / \mathrm{dl}$. La littérature rapporte des moyennes de $8 \mathrm{~g} / \mathrm{dl}$
Tableau 1. Age moyen, Durée en dialyse, PPID, Etiologies et obtention du poids sec.

\begin{tabular}{|c|c|c|c|}
\hline Patients $(n=40)$ & $\begin{array}{c}\text { Poids sec } \\
\text { critères A } \\
(17,5 \%)\end{array}$ & $\begin{array}{c}\text { Poids sec } \\
\text { critères B } \\
(55 \%)\end{array}$ & $\begin{array}{c}\text { Absence de } \\
\text { Poids sec } \\
(27,5 \%) \\
\end{array}$ \\
\hline $\begin{array}{l}\text { Age moyen } \\
\text { (en Année) }\end{array}$ & 46,71 & 42,86 & 50,18 \\
\hline $\begin{array}{l}\text { Durée moyenne en } \\
\text { dialyse (en Année) }\end{array}$ & 3,71 & 3,23 & 3 \\
\hline $\begin{array}{l}\text { Moyenne de PPID } \\
\text { (en Kg) }\end{array}$ & 2 & 3,02 & 3,09 \\
\hline Etiologies & $\begin{array}{l}\text { NIC }(n=4) ; \\
\text { HIVAN }(n=1) ; \\
\text { PKR }(n=1) ; \\
\text { NV }(n=1)\end{array}$ & $\begin{array}{l}\text { GNC }(n=9) ; \\
\text { NV }(n=8) ; \\
\text { ND }(n=2) ; \\
\text { IRRP }(n=1) ; \\
\text { NIC }(n=1) ; \\
\text { NI }(n=1)\end{array}$ & $\begin{array}{l}\text { NV(4); } \\
\text { NI(3); } \\
\text { GNC(2) ; } \\
\text { ND(1); } \\
\text { NIC(1) }\end{array}$ \\
\hline
\end{tabular}

de taux d'Hb chez les patients dialysés en dehors de tout traitement par agents stimulants l'érythropoïèse comme c'était le cas de nos patients. Cette anémie au départ relativement bien tolérée est en corrélation inverse avec la diminution du débit de filtration glomérulaire [10]. Elle est due surtout à une diminution de la durée de vie des hématies, à l'insuffisance de la sécrétion endocrine d'érythropoiétine (EPO) et aux multiples phénomènes de spoliation sanguine chez ces patients.

Sur les clichés de radiographie du thorax de face, 27,5\% de nos patients avaient une cardiomégalie. Il s'agit d'une des complications cardiovasculaires généralement retrouvées chez les patients hémodialysés. Ces cardiomégalies radiologiques masquent plusieurs types de cardiomyopathies connues pour survenir chez ces patients généralement oligoanuriques, hypertendus et anémiés avec des retentions hydrosodées [11-13].

En termes de prise de poids interdialytique (PPID), la moyenne pour nos patients était de 3,08 kg. Des surcharges hydriques à types d'OMI et d'œdème du visage étaient respectivement retrouvées chez $30 \%$ et 7,5\% des patients. Cette PPID (extrême $5 \mathrm{~kg}$ ) contribue au développement de l'HTA volo-dépendante, car elle était largement supérieure à celle conseillée (soit 2,5 kg) entre 2 séances d'hémodialyse $[14,15]$ et ce malgré les conséquentes moyennes d'UF à 2,93 L (95,1 \% de la moyenne de PPID) dont avaient bénéficié les patients. Toutefois, la moyenne de PPID des patients répondant à nos " critères $A$ » était de $2 \mathrm{~kg}$, donc en dessous de la moyenne de PPID de l'ensemble des patients $(3,08 \mathrm{~kg})$ et renvoie dès lors à l'observation des règles diététiques par certains patients. La PAM/E de nos patients était de 138/84 $\mathrm{mm}$ hg avec $35,5 \%$ de normotendus, pour une $\mathrm{PAM} / \mathrm{S}$ à $129 / 81 \mathrm{~mm}$ hg avec $42,5 \%$ de normotendus.

Ces résultats attestent de l'effet de l'UF sur les chiffres tensionnels de sortie. Toutefois, un certain nombre de patients restent hypertendus malgré une UF adéquate, soit $52,5 \%$.

Selon nos critères d'obtention du poids sec :

- les patients répondant aux «critères $A »(17,5 \%)$ : absence d'HTA entre les séances d'hémodialyse, absence de cardiomégalie radiologique, pas de signe de surcharge 
hydrosodée cliniquement ou radiologiquement décelable, pas de prise de médicament antihypertenseur entre les séances d'hémodialyse ;

- ceux répondant aux «critères B» (55\%) : présence d'HTA entre les séances d'hémodialyse voire une PA normale sous médicament antihypertenseur, absence de cardiomégalie, absence de surcharge hydrosodée cliniquement ou radiologiquement décelable.

- ceux qui n'avaient pas atteint leur poids sec donc ne répondant ni «critères $\mathrm{A}$ ou $\mathrm{B}$ » représentaient 27,5\%.

Ces différents profils de patients en matière de poids sec avaient été rapportés par Bonello $\mathrm{M}$ et al [16] qui, dans leur étude avaient combiné plusieurs paramètres d'appréciation du poids sec dont le volume sanguin, la PA, la fréquence cardiaque (FC) et la bio-impédance. Les mêmes auteurs retrouvaient 4 différents types de profils qui étaient:

- Profil A : patients qui au départ en inflation hydrosodée, avaient atteint leur poids sec avec une PA et une FC normales :

- Profil B : patients au départ en inflation hydrosodée, qui avaient atteint leur poids sec avec une PA stable mais une FC augmentée;

- Profil $C$ : patients qui sont restés en inflation avec une PA et une FC instables :

- Profil D : patients déshydratés, ayant atteint le poids sec avec une PA et une FC stables.

Dans notre série, les patients répondants aux «critères $A$ » avaient une durée moyenne en dialyse plus élevée, suivis respectivement par ceux répondants aux «critères B » puis ceux n'ayant pas atteint leur poids sec et inversement pour la PPID, où les patients répondants aux « critères $A$ » avaient les moindres PPID.

Sur le plan étiologique, une NIC était retrouvée chez 57,14\% ( 4 patients sur 7) des patients répondants aux « critères $A$ », tandis que les GNC et les NV prédominaient chez les patients répondants aux «critères $B$ » soit 77,27 \% (17 patients sur 22). Ces constats renvoyaient au rôle du patient dans la quête de son poids sec (respect des règles diététiques), de la néphropathie sous-jacente voire la durée en dialyse.

Nos résultats et ceux des auteurs [16] montraient que l'établissement d'un poids sec en hémodialyse reste difficile et chaque méthode d'évaluation prise isolément s'avérerait insuffisante.

\section{CONCLUSION}

Une combinaison efficiente de plusieurs paramètres d'appréciation de la surcharge hydrique reste la méthode la plus sure pour s'approcher de ce poids idéal, car aucune méthode définie d'établissement de poids sec n'est encore disponible.

Déclaration d'intérêts: les auteurs ne déclarent aucun conflit d'intérêt en rapport avec cet article.

\section{RÉFÉRENCES}

1. Wizemann V, Wabel P, Chamney P, Zaluska W, Moissl U, Rode C et al. The mortality risk of overhydration in haemodialysis patients. Nephrol Dial Transplant .2009; 24:1574-1579.

2. Onofriescu M, Hogas S, Voroneanu L, Covic A. Methods for estimating "dry weight" in hemodialysis patients. Rev Med Chir Soc Med Nat lasi. 2011; 115(3):742-9.

3. Rout $P$, Sandhu GS, Khattak M, Goldfarb-Rumyantzev A. Advances in volume monitoring in dialysis patients. Minerva Urol Nefrol. 2010; 62(1):13-27.

4. Leypoldt JK, Cheung AK. Evaluating volume status in hemodialysis patients. Adv Ren Replace Ther. 1998; 5(1):64-74.

5. Fenech $M$, Jaffrin MY. Evaluation des méthodes d'aide à la détermination du poids sec des patients hémodialysés par BioImpédance. TBM-RBM25. 2004; 25:218-219.

6. Richard F,Gilbert D. Séminaire d'Uro-Nephrologie XXXV serie2009. Université Paris VI faculté de médecine Pitié Salpetrière faculté de médecine Pierre-Marie Curie, Paris VI.

7. Gidienne $C$, Burnat S. Suivi biologique de l'hémodialyse chronique. AN Biol Clin, 2000 ; 58 :663-671.

8. Roupret M. Urologie\&Néphrologie 3ème édition. Paris : VERNAZOBRES-GREGO, 2009.

9. Joly D. Néphrologie. Paris : VERNAZOBRES-GREGO,2009

10. William MC, Stephen LA, Bolton WK, Sally H, Daniel LL, Linda TK et al The prevalence of anemia in patients with chronic kidney disease Current Medical Research and Opinion .2004Volume 20, Issue 9

11. Levin A. Clinical epidemiology of cardiovascular disease in chronic kidney disease prior to dialysis. Semin Dial. 2003 ; 16(2):101-5.

12. Ulasi II, Ardoise EB, ljma CK. Left ventricular hypertrophy in African Black patients with chronic renal failure at first evaluation. Ethn Dis. 2006 ; 16(4):859-64.

13. Malergue MC. L'échocardiographie chez l'hémodialysé chronique. La Lettre du Cardiologue - n³19 - octobre 1999.

14. Pierre S, Kim SA, Christophe C, Philipe LC. Dialyse renale. 2ème édition. Paris : Masson, 2002.

15. Canaud F. Recommandations européennes de bonnes pratiques de dialyse (EBPD). Nephrol Ther.2008; 4:115-124.

16. Bonello $M$, House AA, Cruz D, et al. Integration of blood volume, blood pressure, heart rate and bioimpedance monitoring for the achievement of optimal dry body weight during chronic hemodialysis. Int J Artif Organs. 2007;30(12):1098-108.

Cet article a été publié dans le " Batna Journal of Medical Sciences » BJMS, l'organe officiel de "I'association de la Recherche Pharmaceutique - Batna»

Avantages à publier dans BJMS :

- Open access : une fois publié, votre article est disponible gratuitement au téléchargement

- Soumission gratuite : pas de frais de soumission, contrairement à la plupart des revues "Open Access »

Possibilité de publier dans 3 langues : français, anglais, arabe Qualité de la relecture : des relecteurs/reviewers indépendants géographiquement, respectant l'anonymat, pour garantir la neutralité et la qualité des manuscrits.

Pour plus d'informations, contacter BatnaJMS@gmail.com ou connectez-vous sur le site de la revue : www.batnajms.com 